\title{
EXAMPLES ON HARMONIC MEASURE AND NORMAL NUMBERS ${ }^{1}$
}

\author{
JANG-MEI WU
}

\begin{abstract}
Suppose that $F$ is a bounded set in $\mathbf{R}^{m}, m \geq 2$, with positive capacity. Add to $F$ a disjoint set $E$ so that $E \cup F$ is closed, and let $D=$ $\mathbf{R}^{m} \backslash(E \cup F)$. Under what conditions on the added set $E$ do we have harmonic measure $\omega(F, D)=0$ ? It turns out that besides the size of $E$ near $F$, the location of $E$ relative to $F$ also plays an important role. Our example, based on normal numbers, stresses this fact.
\end{abstract}

Suppose that $F$ is a bounded set in $\mathbf{R}^{m}, m \geq 2$, with positive capacity. Add to $F$ a disjoint set $E$ so that $E \cup F$ is closed, and let $D=\mathbf{R}^{m} \backslash(E \cup F)$. Under what conditions on the added set $E$ do we have harmonic measure $\omega(F, D)=0$ ? It turns out that besides the size of $E$ near $F$, the location of $E$ relative to $F$ also plays an important role. Our example, based on normal numbers, stresses this fact.

THEOREM. Let $D$ be a bounded domain in $\mathbf{R}^{m}, m \geq 2, F$ be a subset of $\partial D$ with $\Lambda^{m-1}(F)=0$, and $E=\mathbf{R}^{m} \backslash(D \cup F)$. Suppose that $F$ lies also on some quasi-smooth curve $\Gamma$ when $m=2$, on some $\mathrm{BMO}_{1}$ surface $\Gamma$ when $m \geq 3$. And suppose that at each $a \in F, 0<r<\frac{1}{4}$, there is a closed set $T \subseteq E \cap B(a, r)$ so that

$$
\operatorname{capacity}(T) \geq \operatorname{capacity}\left(B\left(0, c_{1} r\right)\right)
$$

and

$$
\operatorname{dist}(T, F)>c_{2} r
$$

also

$$
\operatorname{diam}(T)<c_{2} r / 3 \quad \text { when } m=2,
$$

where $c_{1}$ and $c_{2}$ are constants in $(0,1)$. Then $\omega(F, D)=0$.

By $B(a, r)$ we mean $\left\{x \in \mathbf{R}^{m}:|x-a|<r\right\}$; and by capacity we mean $(m-2)$ capacity if $m \geq 3$, and logarithmic capacity if $m=2$. See [2, 1.XIII] for their properties.

When $m \geq 3,(1)$ is equivalent to

$$
\operatorname{capacity}(T) \geq c \text { capacity } B(0, r) \text {. }
$$

However, when $m=2,(1)$ is more restrictive than $\left(1^{\prime}\right)$.

The surface $\Gamma$ and $\partial D$ are in general distinct; no smoothness condition is imposed on $\partial D$. The problem is very different if $\partial D$ is quasi-smooth or $\mathrm{BMO}_{1}$ (see [4]). When $m \geq 3$, topological properties of $D$ are less important in studying $\omega(F, D)$ :

Received by the editors December 18, 1984.

1980 Mathematics Subject Classification. Primary 30C85, 31A15, 31B05, 31B15.

${ }^{1}$ Research partially supported by the National Science Foundation. 
there exist a topological ball $D$ in $\mathbf{R}^{3}$, a set $F$ lying on $\partial D$ and on a plane, so that $\operatorname{dim}(F)=1$, but $\omega(F, D)>0[\mathbf{9}]$.

In the theorem, we have three conditions on $F$ : (a) it lies on a surface $\Gamma$ with minimum smoothness, (b) $\mathbf{R}^{m} \backslash(D \cup F)$ is big near each point in $F$ in the capacity sense, and (c) $\mathbf{R}^{m} \backslash(D \cup F)$ is untangled from $F$ as in (2). $\Gamma$ cannot be too general, the theorem is not true if $\Gamma$ is a quasi-circle (see [7]) and it is quite clear that (1) in some sense is necessary. We shall show in Example 3 that if (a) and (b) are satisfied, but the part of $\mathbf{R}^{m} \backslash(D \cup F)$ that is big near $F$ is not separated from $F$, we may still have $\Lambda^{m-1}(F)=0$ but $\omega(F)>0$.

The proof shows that the theorem still holds when $F$ lies on a slightly more general surface, namely,

$\Gamma$ is a topological sphere in $\mathbf{R}^{m}$, whose interior $\Omega_{1}$ and exterior $\Omega_{2}$ are both nontangentially accessible domains, and on $\Gamma, \Lambda^{m-1}(E)=$ $0 \Rightarrow \omega\left(E, \Omega_{i}\right)=0$ for $i=1,2$.

See [4] for the definitions of quasi-smooth curves (also called chord-arc), $\mathrm{BMO}_{1}$ surfaces and nontangentially accessible domains, and their relations.

This theorem is an improvement of the one in [9]. There $\mathbf{R}^{m} \backslash D$ satisfies a corkscrew condition; that is, $T$ can be chosen to be a ball in $\mathbf{R}^{m}$ with

$$
\text { radius } T>c r \text {. }
$$

It is also closely related to Theorem 2 in [8] and Theorem 3 in [7] when $m=2$ and $D$ is simply-connected. However, the present theorem does not imply the results in [7 or 8], because, for a set $F$ lying on the boundary of a simply-connected domain, we may not be able to find $T$ so that the conditions (1) and (2) are both fulfilled.

To prove the theorem, we assume that $\Gamma$ satisfies the more general condition (4) and follows the steps in [9]. Because our assumption on the size of $T$ is in terms of capacity, which is weaker than (5), we need a replacement of (5) in terms of harmonic measure, which is sufficient for the proof of the theorem. First, by the countable subadditivity of capacity for $m \geq 3$ and by (3) for $m=2$, we can find $c_{3}, c_{4}$ depending on $m, c_{1}$, and $c_{2}$ only so that, for any $a \in F, 0<r<\frac{1}{4}$, there exists $P \in B(a, r)$ with $\operatorname{dist}(P, F)>3 c_{3} r$ and

$$
\operatorname{capacity}\left(B\left(P, c_{3} r\right) \cap T\right)>\operatorname{capacity}\left(B\left(a, c_{4} r\right)\right) \text {. }
$$

Therefore, for $|Q-P|=2 c_{3} r$,

$$
w^{Q}\left(T \cap \overline{B\left(P, c_{3} r\right)}, B\left(P, 3 c_{3} r\right) \backslash\left(T \cap \overline{B\left(P, c_{3} r\right)}\right)\right)>c_{5}>0 .
$$

We remark that when $m=2$, these statements do not follow from $\left(1^{\prime}\right)$. The rest of the proof follows the similar lines as in [9].

Here are some applications.

EXAMPLE 1. In $\mathbf{R}^{m}$, there exist disjoint sets $E$ and $F$ in $B \overline{(0, \sqrt{m})}$, whose union $E \cup F$ is closed, of zero $\Lambda^{m-1}$-measure, and each of which has positive capacity. However, $\omega(E, D)>0, \omega(F, D)=0$ where $D=B(0, m) \backslash(E \cup F)$. Moreover, for any $\beta, \alpha$ in $[m-2, m-1], E$ and $F$ can be chosen with $\operatorname{dim} E=\beta$ and $\operatorname{dim} F=\alpha$; in particular, it is possible to have $\operatorname{dim} E=m-2, \operatorname{dim} F=m-1$.

We give the construction for $m=3$; some necessary changes are needed for other $m$ 's. Given a sequence $\left\{r_{n}\right\}_{1}^{\infty} \supseteq\left(0, \frac{1}{2}\right)$, we construct the corresponding Cantor set 
$S$ : Let $S_{0,1}=[0,1] ; S_{1,1}$ and $S_{1,2}$ be the two closed intervals of length $r_{1}$ each, left after the middle $\left(1-2 r_{1}\right)$ portion of $[0,1]$ is removed. At the $n$th step, let $\left\{S_{n, k}\right\}_{k=1}^{2^{n}}$ be the closed intervals of length $\prod_{1}^{n} r_{j}$ each, left after the middle $\left(1-2 r_{n}\right)$ portion of each $\left\{S_{n-1, k}\right\}_{1}^{2^{n-1}}$ is removed. Let

$$
S=\bigcap_{1}^{\infty} \bigcup_{k=1}^{2^{n}} S_{n, k} .
$$

Let $\left\{A_{n, l}\right\}_{l=1}^{4^{n}}=\left\{S_{n, k} \times S_{n, j}: 1 \leq k, j \leq 2^{n}\right\}$ and $A=\bigcap_{n=1}^{\infty}\left(\bigcup_{l=1}^{4^{n}} A_{n, l}\right)$ the Cantor set on $[0,1] \times[0,1]$. We choose

$$
r_{n}= \begin{cases}\frac{1}{4}\left(\frac{n+3}{n+2}\right)^{2}, & \alpha=1 \\ 4^{-1 / \alpha}, & 1<\alpha<2 \\ \frac{1}{2} \frac{n}{n+1}, & \alpha=2 .\end{cases}
$$

Standard calculation shows that $\operatorname{dim} A=\alpha, \Lambda^{2}(A)=0$ and 1-capacity $(A) \equiv$ $c_{\alpha}>0$. We let $F$ be $A \times\{0\}$ in $R^{3}$.

Following the construction of $A$, we may construct a Cantor set $E_{n, l}$ in $A_{n, l} \times$ $\left\{\prod_{1}^{n} r_{j}\right\} \subseteq \mathbf{R}^{2} \times\left\{\prod_{1}^{n} r_{j}\right\} \subseteq \mathbf{R}^{3}$ satisfying $\operatorname{dim} E_{n, l}=\beta, \Lambda^{2}\left(E_{n, l}\right)=0$ and

$$
\operatorname{capacity}\left(E_{n, l}\right)=c_{\beta} \times \prod_{1}^{n} r_{j} .
$$

Let $E=\bigcup_{n=1}^{\infty}\left(\bigcup_{l=1}^{4^{n}} E_{n, l}\right)$, thus $\operatorname{dim} E=\beta, \Lambda^{2}(E)=0$ and capacity $(E)>0$. Let $D=B(0, m) \backslash(E \cup F)$. Because of $(7)$, we see that, at each $a \in F$, for $\sqrt{m} \prod_{1}^{n} r_{j} \leq$ $r<\sqrt{m} \prod_{1}^{n-1} r_{j}$, there exists some $E_{n, l}$ so that

$$
\operatorname{dist}\left(E_{n, l}, A\right)>c r \quad \text { and } \operatorname{capacity}\left(E_{n, l}\right)>c r .
$$

From the theorem, it follows that $\omega(F, D)=0$. Since

$$
\operatorname{capacity}(E \cup F)>0 \text {, }
$$

we must have $\omega(E, D)>0$.

EXAMPLE 2. Let

$$
r_{n}= \begin{cases}(n+2)^{-1}, & \alpha=0, \\ 2^{-1 / \alpha}, & 0<\alpha<1, \\ n / 2(n+1), & \alpha=1 .\end{cases}
$$

Let $\alpha_{1}$ and $\alpha_{2}$ be numbers in [0,1], not both zero, and $S_{j}, j=1,2$, be the Cantor set on $[0,1]$ corresponding to $\left\{r_{n}\right\}$ with $\alpha=\alpha_{j}$. Clearly, $\operatorname{dim} S_{j}=\alpha_{j}$. Let $D=$ $\mathbf{R}^{2} \backslash\left(S_{1} \times S_{2}\right)$ be the domain in $\mathbf{R}^{2}$, whose boundary is the planar Cantor set $S_{1} \times S_{2}$ of dimension $\alpha_{1}+\alpha_{2}$. Then any line parallel to either coordinate axis meets $\partial D$ on a set of zero harmonic measure and of dimension $\alpha_{1}$ or $\alpha_{2}$. The example is particularly interesting when $\alpha_{1}=0$ (or $\alpha_{2}=0$ ).

Some complementary examples on domains whose boundaries consist of other Cantor sets can be found in [8]. 
The next example shows that condition (2)-ensuring that $E$ and $F$ are untangled-is not superfluous.

The author thanks R. Kaufman for bringing the concept of normal numbers to her attention.

EXAMPLE 3. In $\mathbf{R}^{m}$, there exist disjoint sets $E$ and $F$ in $B(0, \sqrt{m}) \cap\left(R^{m-1} \times\right.$ $\{0\})$, with the properties that each set has zero $\Lambda^{m-1}$-measure, positive capacity, that $E \cup F$ is closed, and that, for any $a \in F, 0<r<\frac{1}{4}$,

$$
\operatorname{capacity}(B(a, r) \cap E)>\text { capacity } B(0, c r) \text {. }
$$

However, $w(F, D)>0$, where $D=B(0, m) \backslash(E \cup F)$. Moreover, for any $\alpha \in$ $(m-2, m-1]$, we can choose $E$ and $F$ so that $\operatorname{dim}(E \cup F)=\alpha$.

We first consider the case $m=3$ and $1<\alpha \leq 2$, and let $r_{n}$ be as in (6), $S$ and $A$ be the Cantor sets on $[0,1]$ or on $[0,1] \times[0,1]$ as defined in Example 1, and let $D=B(0,3) \backslash A$. We identify $\mathbf{R}^{2}$ with $\mathbf{R}^{2} \times\{0\}$.

We represent a number $a \in S$ by $\left(a_{1}, a_{2}, a_{3}, \ldots\right)$, where $a_{n}=0$ if $a$ is in the left half of some interval $S_{n-1, k}$, and $a_{n}=1$ if $a$ is in the right half. We define $f: S \rightarrow[0,1]$ by

$$
f(a)=\sum_{1}^{\infty} \frac{a_{n}}{2^{n}} .
$$

A number $a$ in $[0,1]$ is called simply normal in the scale of 2 if its binary expansion $\sum_{1}^{\infty}\left(a_{n} / 2^{n}\right)$ has the property that

$$
n^{-1} \sum_{j=1}^{n} a_{j} \rightarrow \frac{1}{2} \quad \text { as } n \rightarrow \infty .
$$

It is known $[\mathbf{1}$ and $\mathbf{3}$, p. 124] that Lebesgue-almost every point in $[0,1]$ is simply normal in the scale of 2 . We let

$$
N=\{a \in S: f(a) \text { is simply normal in the scale of } 2\},
$$

$\beta$ be a number in $\left(0, \frac{1}{2}\right)$ which satisfies

$$
1-\beta<2^{-1 / \alpha}
$$

and $M=\left\{a \in S: n^{-1} \sum_{1}^{r} a_{j} \rightarrow \beta\right.$, as $\left.n \rightarrow \infty\right\}$.

Because $N \times N$ has Lebesgue measure 1 ,

$$
\operatorname{capacity}(N \times N)>0 \text {. }
$$

We claim that

$$
\operatorname{capacity}(M \times M)>0 \text {. }
$$

Assuming this is true, we proceed as follows. Because $r_{n}$ are constant for $1<\alpha<2$ and $r_{n}$ are increasing for $\alpha=2$, by an appropriate scaling, we obtain, for any $a \in A$, $0<r<1$,

$$
\begin{aligned}
& \operatorname{capacity}(N \times N \cap B(a, r))>c r \\
& \operatorname{capacity}(M \times M \cap B(a, r))>c r .
\end{aligned}
$$


Since capacity $(A)>0$, we have $\omega(A, D)>0$. Therefore, at least one of the three sets, $N \times N, M \times M$, or $A \backslash(N \times N \cup M \times M)$ has positive harmonic measure with respect to $D$.

Question. Which one?

We choose $F$ to be any one of these three which has positive harmonic measure, and $E=A \backslash F$. Then (8) follows from (12) or (13).

To prove (11), we define a measure $\lambda$ on $[0,1]$ by

$$
\lambda(I)=\prod_{j=1}^{n}\left[(2 \beta-1) a_{j}+1-\beta\right]
$$

if $I$ is the closed interval

$$
\left[\sum_{1}^{n} \frac{a_{j}}{2^{j}}, \frac{1}{2^{n}}+\sum_{1}^{n} \frac{a_{j}}{2^{j}}\right]
$$

for any $n>0$ and $a_{j}=0$ or 1 , and let $\nu$ be the induced measure on $S, \nu(E)=$ $\lambda(f(E))$.

It is clear that $\nu([0,1])=1$.

We need to show that

$$
\nu(M)=1 \text {. }
$$

Let $X_{j}$ be random variables on $([0,1], \lambda)$ so that $X_{j}(a)=a_{j}$. They are independent, uniformly bounded and $E\left(X_{j}\right)=\beta$. It is proved in [6] by using ideas from $[5, \mathrm{p}$. 131] that

$$
\sum_{1}^{n} X_{j}-n \beta=o\left(n^{2 / 3}\right), \quad n \rightarrow \infty
$$

for $\lambda$-almost every $a$ in $[0,1]$. This says that $\lambda(f(M))=1$ or $\nu(M)=1$.

Because of (14),

$$
\nu \times \nu\left(M \times M \cap B\left(P, \prod_{j=1}^{n} r_{j}\right)\right) \leq 4(1-\beta)^{2 n}
$$

for any $P \in \mathbf{R}^{3}$. With the aid of (6) and (9) we conclude

$$
\begin{aligned}
\int_{M \times M}|P-Q|^{-1} d \nu \times \nu(Q) \\
\leq C\left[\frac{1}{1-2 r_{1}}+\sum_{n=1}^{\infty} \frac{4}{\left(\prod_{j=1}^{n} r_{j}\right)\left(1-2 r_{n+1}\right)}(1-\beta)^{2 n}\right]<C_{\alpha, \beta}<+\infty
\end{aligned}
$$

for any $P \in \mathbf{R}^{3}$. This, together with (15), shows that $M \times M$ has positive capacity.

(10) can also be obtained by replacing $\beta$ by $\frac{1}{2}$ in the proof above. This completes the case $m=3$. To arrive at the example for $m \geq 4$, we need only some routine changes.

An alternative way to show (11) is calculating the Hausdorff dimension of $M \times M$. This approach is particularly convenient for $m=2$, when the logarithmic potential is harder to estimate. 
When $m=2,0<\alpha \leq 1$, we let

$$
r_{n}= \begin{cases}2^{-1 / \alpha}, & 0<\alpha<1, \\ n / 2(n+1), & \alpha=1,\end{cases}
$$

and construct the Cantor set $S$ of dimension $\alpha, M$ and $N$, accordingly. Combining results in $[\mathbf{1}$ and $\mathbf{6}]$, we see that

$$
\operatorname{dim} f(M)=\frac{\beta \log \beta+(1-\beta) \log (1-\beta)}{\log \frac{1}{2}} .
$$

By translating coverings of $M$ through $f$, we can show that

$$
\operatorname{dim} M=\alpha[\beta \log \beta+(1-\beta) \log (1-\beta)] / \log \frac{1}{2}>0 .
$$

Since $f(N)$ are the simply normal numbers, $\operatorname{dim} f(N)=1$ and $\operatorname{dim} N=\alpha$. Now let $A \equiv S$, and $F$ be any one of the three sets $N, M$ and $A \backslash(N \cup M)$ which has positive harmonic measure with respect to $D \equiv B(0,2) \backslash A$. The properties in the example are satisfied.

Question. We do not know whether $A$ can be chosen in Example 3 to have $\operatorname{dim} A=m-2$. When $m=3, \alpha=1,\left\{r_{n}\right\}$ is not increasing, thus (12) does not follow from (10) automatically, and we do not even know whether $r_{n}$ can be chosen so that capacity $(A \cap B(a, r))>c r$ for all $a \in A, 0<r<\frac{1}{4}$.

REMARK. When $m \geq 3$, the domain $D$ in Example 3 can be made into a topological ball, by deleting a branching tree $T$, connecting $B(0, m)$ to $A$, from $B(0, m) \backslash A$. This is possible because line segments have capacity zero when $m \geq 3$. The branches of $T$ should be chosen carefully so that the ratio of capacity $(T)$ to $\operatorname{capacity}(A)$ is sufficiently small.

\section{REFERENCES}

1. A. S. Besicovitch, On the sum of digits of real numbers represented in the dyadic system. (On sets of fractional dimensions. II), Math. Ann. 110 (1935), 321-330.

2. J. L. Doob, Classical potential theory and its probabilistic counterpart, Springer-Verlag, Berlin and New York, 1983.

3. G. H. Hardy and E. M. Wright, An introduction to the theory of numbers (5th ed.), Claredon, Oxford, 1979.

4. D. S. Jerison and C. E. Kenig, Boundary behavior of harmonic functions in non-tangentially accessible domains, Adv. in Math. 46 (1982), 80-147.

5. S. Kaczmarz and H. Steinhaus, Theorie der Orthogonalreihen, PWN, Warsaw, 1935.

6. R. Kaufman, A further example on scales of Hausdorff functions, J. London Math. Soc. (2) 8 (1974), 585-586.

7. R. Kaufman and J.-M. Wu, Distortion of the boundary under conformal mapping, Michigan Math. J. 29 (1982), 267-280.

8. B. Øksendal, Brownian motion and sets of harmonic measure zero, Pacific J. Math. 95 (1981), 193-204.

9. J.-M. Wu, On singularity of harmonic measure in space, Pacific J. Math. (to appear).

DEPARTMENT OF MATHEMATICS, UNIVERSITY OF ILLINOIS, 1409 WEST GREEN STREET, URBANA, ILLINOIS 61801 